\title{
Single-component surface in binary self-assembled NaK nanoalloy clusters
}

\author{
M. Tchaplyguine, ${ }^{1, *}$ S. Legendre, ${ }^{2}$ A. Rosso, ${ }^{2}$ I. Bradeanu, ${ }^{2}$ G. Öhrwall, ${ }^{1}$ S. E. Canton, ${ }^{3}$ T. Andersson, ${ }^{2}$ N. Mårtensson,,${ }^{1,2}$ \\ S. Svensson, ${ }^{2}$ and O. Björneholm ${ }^{2}$ \\ ${ }^{1}$ MAX-Laboratory, Lund University, P.O. Box 118, 22100 Lund, Sweden \\ ${ }^{2}$ Department of Physics, Uppsala University, P.O. Box 530, 75181 Uppsala, Sweden \\ ${ }^{3}$ Department of Chemical Physics, Lund University, P.O. Box 118, 22100 Lund, Sweden
}

(Received 13 May 2009; published 7 July 2009)

\begin{abstract}
Free metallic nanoalloy clusters created in a self-assembling process out of sodium and potassium mixed vapor have been studied by synchrotron-based photoelectron spectroscopy. The clusters are shown to consist of an alloy core surrounded by a surface layer containing only $\mathrm{K}$ in a range of conditions from K-rich to Na-rich nanoalloys. The size of the clusters as well as the fraction of the elements has been estimated from the spectra using our results on pure clusters. The mechanism behind the observed structure is discussed in terms of the total cohesive-energy minimization.
\end{abstract}

DOI: 10.1103/PhysRevB.80.033405

PACS number(s): 79.60.-i, 64.75.Jk, 64.75.Op, 64.75.Yz

Nanoalloys are metallic clusters composed of two or more elements. ${ }^{1}$ Properties of such complex nanoparticles are defined not only by their size and stoichiometry but also by the internal spatial distribution of the components. Examples of different spatial distributions are random mixtures, ordered compounds, radially segregated structures, and core-shell structures, in which an outer layer of one composition surrounds the inner core of another composition. Due to the rich prospects for tailoring properties offered by control of size, stoichiometry, and spatial distribution, nanoalloys are promising candidates as functional building blocks for nanomaterials. ${ }^{1}$ To realize this, understanding and control of the cluster-formation process as well as accurate characterization methods are needed.

The type of spatial distribution in $A_{x} B_{y}$ nanoalloys depends on several, partly interconnected, factors, of which many are common also to macroscopic alloys. ${ }^{1}$ The most important factor is the relative strengths of the $A-A$, the $B-B$, and the $A-B$ bonds. In the first approximation, if $A-B$ bonds are the strongest, mixing is favored; otherwise, segregation is favored. In the latter case, the segregation is primarily determined by the relative surface energies of components $A$ and $B$ with the component with the lowest surface energy segregated to the surface. Another important factor is the relation between the component atomic sizes: larger atoms tend to occupy surface sites, which are less confined than bulk ones. The spatial distribution of the components for a particular $A-B$ nanoalloy depends critically on the balance between these factors as well as on the cluster-formation process. ${ }^{1}$ While some formation processes may result in stable lowenergy structures, other formation processes may result in various metastable structures, opening the possibility of intracluster nanoengineering.

$\mathrm{NaK}$ is an alloy of sodium $(\mathrm{Na})$ and potassium $(\mathrm{K})$. The two metals are freely miscible in all proportions, and in a wide composition range the alloy is liquid at room temperature. For the $\mathrm{NaK}$ system, Na has higher cohesive energy than $\mathrm{K}$ (1.11 and $0.93 \mathrm{eV}$, respectively) and the atomic size of $\mathrm{K}$ is $\approx 25 \%$ larger than that of $\mathrm{Na}$. Both the main factors influencing the component spatial distribution thus favor $\mathrm{K}$-surface enrichment for the $\mathrm{NaK}$ system. For macroscopic $\mathrm{NaK}$ alloys, theoretical calculations based on density- functional theory ${ }^{2}$ and $a b$ initio molecular-dynamics simulations $^{3}$ predict $\mathrm{K}$ to be enriched at the surface. For $\mathrm{K}$-rich alloys, the surface may be almost completely dominated by $\mathrm{K}$, and this domination decreases with increasing $\mathrm{Na}$ content and temperature. ${ }^{2}$ On the opposite side of the dimensional scale, calculations using density-functional theory predict $\mathrm{NaK}$ clusters of equal $\mathrm{Na} / \mathrm{K}$ proportions consisting of tens of atoms to have ground-state structures at $0 \mathrm{~K}$ with a strongly K-dominated surface. ${ }^{4}$ None of these theoretical predictions have to the best of our knowledge been tested experimentally. We will here present an experimental study of NaK nanoalloy clusters produced by gas aggregation. Using the elemental and site sensitivity of $\mathrm{X}$-ray photoelectron spectroscopy (XPS) based on synchrotron radiation (SR), we will determine the cluster composition and the size, and especially address the question whether the predicted surface enrichment of $\mathrm{K}$ is observed even when the clusters are formed by gas aggregation, i.e., random aggregation of $\mathrm{Na}$ and $\mathrm{K}$ atoms to a hot growing cluster, which is rapidly cooled down to $\approx 100 \mathrm{~K}$. This will be done not only for the case of a K-rich alloy core, as in the calculations, but also for the case of a Na-rich alloy core.

The experiments have been performed at MAXLaboratory, Lund, Sweden, using the I411 beamline equipped with a Scienta R4000 electron spectrometer. The homebuilt cluster source, based on the gas-aggregation principle, ${ }^{5,6}$ has earlier showed the capability to produce a beam of alkali-metal clusters containing thousands of atoms with concentrations high enough to be probed by SR-based XPS. ${ }^{7,8}$ Although this type of cluster sources creates a certain distribution of sizes, the corresponding spread of the binding energies is insignificant in the cluster-size range in question. In the present work the cluster source consisted of a furnace placed inside a liquid-nitrogen-cooled cryostat with a continuous flow of Ar through it. The Na-K alloy was prepared in the furnace, by first loading it with solid $\mathrm{Na}$ and $\mathrm{K}$ in $\approx 1: 2$ mass or $\approx 1: 1$ mole proportion and then by slow melting of the load under vacuum. For the working furnace temperature $\left(\sim 300{ }^{\circ} \mathrm{C}\right)$ the vapor pressure of $\mathrm{K}$ is several times higher than that of $\mathrm{Na}^{9}{ }^{9}$ The $\mathrm{Na} 2 p$ and the $\mathrm{K} 3 p$ binding-energy regions have been probed by XPS from the moment a stable working temperature was reached until the 


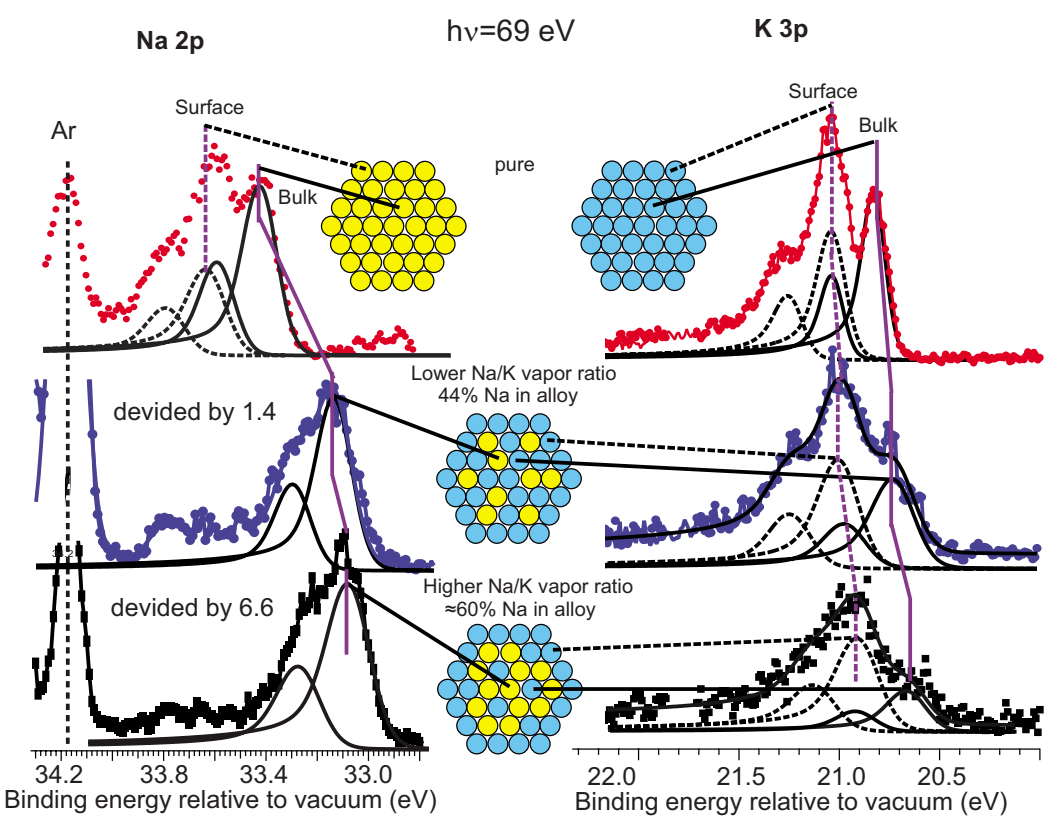

FIG. 1. (Color online) Na $2 p$ and K $3 p$ XPS spectra for pure single-component $\mathrm{Na}$ and $\mathrm{K}$ clusters (top), clusters produced from the vapor of lower $\mathrm{Na} / \mathrm{K}$ ratio (middle), and clusters produced from the vapor of higher $\mathrm{Na} / \mathrm{K}$ ratio (bottom). For the two mixed cases, the $\mathrm{Na} 2 p$ and the K $3 p$ spectra are parts of the same spectra, from which the two regions have been extracted. For presentational clarity, the Na signal is divided by a factor of 1.4 in the middle spectrum and by 6.6 in the bottom spectrum relative to the corresponding $\mathrm{K}$ signal. An $\operatorname{Ar} 3 p^{4} n l$ satellite line is seen above the $\mathrm{Na} 2 p$ features. load in the furnace was totally evaporated. By recording snapshot core-level spectra, we could see that the relative $\mathrm{K} / \mathrm{Na}$ vapor ratio, and thus the clustering conditions, changed insignificantly for an extended period of time once the furnace working temperature had been reached. The higher vapor pressure of $\mathrm{K}$ led to a quicker consumption of $\mathrm{K}$ relative to $\mathrm{Na}$. Close to the very end of the furnace load, this caused a gradual increase in $\mathrm{Na} / \mathrm{K}$ vapor ratio, resulting in a change in the relative $\mathrm{Na} / \mathrm{K}$ cluster composition ratio. The spectra were recorded using $69 \mathrm{eV}$ photon energy. The presence of Ar lines in the spectrum, due to Ar atoms within the cluster beam, allowed precise absolute binding-energy calibration relative to the vacuum level and determination of the total instrumental broadening $(\sim 0.12 \mathrm{eV})$.

Figure 1 presents typical XPS spectra of the $\mathrm{Na} 2 p$ and the $\mathrm{K} 3 p$ regions for pure single-component $\mathrm{Na}$ and $\mathrm{K}$ clusters (top), snapshot spectra for the clusters produced from the vapor of lower $\mathrm{Na} / \mathrm{K}$ ratio (middle), and for the clusters produced from the vapor of higher $\mathrm{Na} / \mathrm{K}$ ratio (bottom), together with schematic illustrations of the deduced cluster structures. We will start our discussion of the NaK clusters by step-bystep demonstrating that the clusters in the case of mixedvapor aggregation are not separated $\mathrm{Na}$ and $\mathrm{K}$ clusters but indeed alloy $\mathrm{NaK}$ clusters.

To understand the mixed-case spectra, we first turn to the pure-case spectra. Each of the pure-case spectra consists of two $p_{3 / 2,1 / 2}$ doublets, all together four peaks. For both $\mathrm{Na}$ and $\mathrm{K}$, the doublet at higher binding energy corresponds to the ionization of the surface atoms and the one at lower binding energy to the ionization of the bulk atoms. We will first consider the mixed case with the low $\mathrm{Na} / \mathrm{K}$ vapor ratio (Fig. 1, middle panel). In this case the shape of the $\mathrm{K} 3 p$ spectrum is rather different from the pure case but, as indicated, still requires two $p_{3 / 2,1 / 2}$ doublets to be well fitted. The subcomponents in the mixed case are shifted down by $\approx 0.1 \mathrm{eV}$ relative to the pure $\mathrm{K}$ case. Another observation for the mixed case is that the bulk/surface ratio is noticeably lower than in the pure case. If we temporarily assume that the sample in the mixed case would not be alloy clusters but pure $\mathrm{K}$ clusters, we note that while the downward shift in energy would imply larger clusters, ${ }^{7,8}$ the smaller bulk/ surface ratio would instead imply smaller clusters. This contradiction shows that the clusterized $\mathrm{K}$ atoms in the mixed case are not a part of pure $\mathrm{K}$ clusters.

The most striking difference between the spectra in the pure and mixed cases is seen in the $\mathrm{Na} 2 p$ spectrum. The binding energy of the mixed-case $\mathrm{Na} 2 p$ feature is $\approx 0.4 \mathrm{eV}$ lower than that for the pure $\mathrm{Na}$ clusters produced at similar conditions (if the centers of gravity are compared). The mixed-case feature also differs in spectral shape relative to that of the pure case. As seen in the top panel, the pure Na-cluster spectrum consists of two $p_{3 / 2,1 / 2}$ doublets: one due to the surface and one due to the bulk atoms. ${ }^{7}$ In contrast, the mixed-case feature is well described by only one $p_{3 / 2,1 / 2}$ doublet. This shows that the probed $\mathrm{Na}$ atoms do not occupy both surface and bulk sites, as in the pure clusters, but only one of these types of sites. When we combine this with the discussed above observation from the $\mathrm{K}$ spectrum-that the clusters are relatively large but that the fraction of $\mathrm{K}$ atoms located in the bulk is low-we are led to a structural model of the clusters with a surface consisting of only $\mathrm{K}$ and a core, being a mixture of $\mathrm{K}$ and $\mathrm{Na}$.

We will now proceed to test our structural model against the differences in binding energies between the cluster and the solid cases. We note that there is a substantial downward binding-energy shift for the mixed clusters for $\mathrm{Na}$ $(\approx 0.4 \mathrm{eV})$ relative to the pure case but only a minor shift for $\mathrm{K}(\approx 0.1 \mathrm{eV})$. Starting with $\mathrm{Na}$, we then first have to put the cluster and solid cases on a common binding-energy scale, namely, absolute binding energies, $E_{\mathrm{vac}}$, measured relative to the vacuum level. The binding energies for grounded solids, $E_{\text {sol,F, }}$ are commonly referred to the Fermi level, and the energy of the Fermi level relative to the vacuum level is the work function $W_{\text {sol }}$ :

$$
E_{\mathrm{sol}, \mathrm{vac}}=E_{\mathrm{sol}, \mathrm{F}}+W_{\mathrm{sol}} .
$$


On an absolute-energy scale, the $\mathrm{Na} 2 p_{3 / 2}$ binding energy for the supported bulk is $33.45 \pm 0.1 \mathrm{eV} \cdot{ }^{10-12}$ In the present case the clusters can be treated as metallic spheres with radius $R$, for which the binding energies $E_{\mathrm{cl} \text {,vac }}$ should be larger than $E_{\text {sol,vac }}{ }^{13}$

$$
E_{\mathrm{cl}, \mathrm{vac}}=E_{\mathrm{sol}, \mathrm{vac}}+\alpha e^{2} / R, \alpha \approx \frac{1}{2} .
$$

The experimental $E_{\mathrm{cl}, \mathrm{vac}}$ is $33.10 \mathrm{eV}$, which, however, would mean that $E_{\mathrm{cl} \text {,vac }}<E_{\mathrm{sol} \text {,vac }}$, in contradiction to Eq. (2). To resolve this we need to further take into account the influence of the work function $W$. Work-function lowering has been observed in the case of $\mathrm{K}$ adsorbed on a metallic substrate. ${ }^{14}$ There it has been shown that one monolayer was enough to decrease the work function of the K/Ag system to the value for pure $\mathrm{K}$. In the system where the bulk is an alloy of $\mathrm{Na}$ and $\mathrm{K}$ and $\mathrm{K}$ dominates the surface, the $\mathrm{Na} 2 p$ binding energy $E_{\text {sol,vac }}$ can be estimated using Eq. (1). $E_{\text {sol,F }}$ would as above be the value for solid $\mathrm{Na}$ (Ref. 10) but the work function would be for $\mathrm{K}$. $\mathrm{K}$ has about $0.5 \mathrm{eV}$ lower work function $(\approx 2.3 \mathrm{eV})$ than $\mathrm{Na}(2.8 \mathrm{eV}) .{ }^{11}$ The absolute bulk Na binding energy, $E_{\text {sol,vac }}$, would thus be decreased by $\approx 0.5 \mathrm{eV}$, which makes it $\approx 0.25 \mathrm{eV}$ lower than the experimentally observed $\mathrm{Na} 2 p$ binding energy for the mixed-case clusters. We obtain $E_{\mathrm{cl}}>E_{\text {sol,vac }}$, in agreement with Eq. (2) and can thus resolve the contradiction for the $\mathrm{Na} 2 p$ level. We can now turn to the $\mathrm{K} 3 p$ bulk signal. It exhibits only a minor shift since the $\mathrm{K}$ bulk-atom energies are not affected, as in both pure and mixed cases the $\mathrm{K}$ surface determines the work function. The minor shift can instead be attributed to the changed local bulk environment due to the alloying, and possibly also to the difference in the cluster size. These factors would also make a minor contribution to the larger shift observed for $\mathrm{Na}$. We conclude that the experimentally observed surface/ bulk ratio and binding energies of the clusters in the case of mixed-vapor aggregation are inconsistent with separate $\mathrm{Na}$ and $\mathrm{K}$ clusters but are well explained if the clusters are mixed $\mathrm{NaK}$ clusters with a $\mathrm{K}$ surface layer surrounding a $\mathrm{NaK}$ alloy core, corroborating the theoretical predictions.

Having established the overall structure of the clusters, we will now proceed to a more detailed characterization of the clusters in terms of the composition and the size. It has to be borne in mind that the gas-aggregation process used here does not produce clusters of one unique size and composition but a distribution of sizes and compositions. The extracted values for the composition and the size therefore have to be understood as effective values of the produced distribution.

To estimate the average size, we will compare the relative bulk and surface signal intensities. To do this we have to compare the $\mathrm{K}$ surface signal to the total bulk signal, i.e., the sum of the $\mathrm{K}$ and the Na bulk signals. We note that since the corresponding $\mathrm{Na}$ and $\mathrm{K}$ mixed-cluster spectra of Fig. 1 are actually a part of the same snapshot spectrum, the photon flux and recording time are identical. After dividing the observed $\mathrm{Na}$ bulk intensity with the relative ionization cross section of the Na $2 p$ and $\mathrm{K} 3 p$ levels $\left(\sigma_{\mathrm{N} \alpha}: \sigma_{\mathrm{K}} \approx 7\right),{ }^{15}$ we can add thus normalized $\mathrm{Na}$ intensity to the $\mathrm{K} 3 p$ bulk intensity. The total bulk signal, including both $\mathrm{Na}$ and $\mathrm{K}$ contributions, is then found to be 1.0 and 1.3 times the surface signal for the middle and for the bottom cases (Fig. 1) correspondingly.
The spectral bulk/surface intensity ratio depends on the sizedependent ratio between the bulk and the surface sites in the clusters and on the relative attenuation of the bulk signal due to the finite electron mean-free paths. ${ }^{16} \mathrm{We}$ have earlier recorded such bulk/surface intensity ratios for both pure $\mathrm{Na}$ and pure $\mathrm{K}$ clusters at similar photon energies. ${ }^{7,8}$ By comparing with these works, where a quasispherical shape of the clusters was used for modeling the bulk/surface intensity, we arrive at an estimate of the average size of the mixed $\mathrm{NaK}$ clusters in the range of $10^{3}-10^{5}$ atoms, implying a diameter in the range from several $\mathrm{nm}$ to $\sim 10^{2} \mathrm{~nm}$. We note that even though this size estimate is crude (to a great extent due to the spread in the published values for the electron mean-free paths), it validates the above assumption made in connection to Eqs. (1) and (2) of the clusters being microscopic metal spheres.

To estimate the composition, i.e., the $\mathrm{Na} / \mathrm{K}$ ratio, we note that according to the calculations for the $\mathrm{NaK}$ liquid alloy the bulk-composition ratio reaches the value of the initial mixing proportion already in the second subsurface layer, with only a minor deviation for the first subsurface layer. ${ }^{3}$ Based on this, we consider the bulk of the clusters to be homogenously mixed. We can then use the observed $\mathrm{Na} / \mathrm{K}$ bulk signal intensity ratio to estimate the stoichiometry of the cluster core. This ratio, however, is also influenced by the ionization cross section, as discussed above, and the different surface sensitivities of the $\mathrm{Na} 2 p$ and $\mathrm{K} 3 p$ signals. The latter effect arises since the $\mathrm{Na} 2 p$ and the $\mathrm{K} 3 p$ levels have different binding energies, resulting in different kinetic energies and consequently different surface sensitivities due to the variation in the electron inelastic mean-free path. In the present case, both signals come out with kinetic energies close to the minimum in the electron inelastic mean-free path, where its variation is small, so we consider the difference in surface sensitivity of the $\mathrm{Na} 2 p$ and the $\mathrm{K} 3 p$ signals to be negligible. With these points in mind, we can estimate the stoichiometry of the alloy core. The experimentally observed $\mathrm{Na} / \mathrm{K}$ bulk intensity ratio for the case of lower $\mathrm{Na} / \mathrm{K}$ vapor ratio (Fig. 1, middle panel) is $\approx 5.5$. This corresponds to an alloy of approximately $\mathrm{Na}_{0.44} \mathrm{~K}_{0.56}$ composition in the core. The mixed $\mathrm{NaK}$ clusters produced with lower $\mathrm{Na} / \mathrm{K}$ vapor ratio thus consist of an alloy core with approximately equal amounts of $\mathrm{Na}$ and $\mathrm{K}$, surrounded by a layer of $\mathrm{K}$. This structure is consistent with the predictions for small, mixed $\mathrm{Na}_{0.5} \mathrm{~K}_{0.5}$ clusters ${ }^{4}$ and liquid K-rich alloys, ${ }^{3}$ whereas liquid alloys of the observed composition have been predicted to have a surface with a small but non-negligible $\mathrm{Na}$ fraction. ${ }^{2}$

For increasingly Na-rich macroscopic alloys, theory indicates a gradually decreasing $\mathrm{K}$ surface dominance. ${ }^{2}$ As stated above the $\mathrm{Na} / \mathrm{K}$ fraction in the vapor increased close to the end of the furnace load, resulting in a correspondingly changed composition of the clusters. Using such a situation, we have probed a more Na-rich case. The spectra shown in the lower panel of Fig. 1 exhibit a $\mathrm{Na} / \mathrm{K}$ bulk intensity ratio of $\approx 11$, i.e., twice as high as for the low $\mathrm{Na} / \mathrm{K}$ vapor-ratio case. We obtain that the alloy core of the high $\mathrm{Na} / \mathrm{K}$ vaporratio case consists of $\mathrm{Na}_{0.61} \mathrm{~K}_{0.39}$, i.e., a Na-dominated core in contrast to the K-dominated core for the low $\mathrm{Na} / \mathrm{K}$ vaporratio case. The spectral shapes are qualitatively very similar to the low $\mathrm{Na} / \mathrm{K}$ vapor-ratio case, implying a similar struc- 
ture with an alloy core surrounded by a layer of $\mathrm{K}$. This means that the surface segregation of $\mathrm{K}$ occurs in a range of core compositions from approximately equal amounts to $\mathrm{Na}$ rich. The surface may consist of one atomic layer of $\mathrm{K}$ even when $\mathrm{K}$ is the minority species in the alloy core.

The driving force behind the observed surface-segregated core-shell structure is energy minimization. Atoms at the surface have lower coordination than those in the bulk, leading to a reduced cohesive energy for the surface atoms. In a mixed system, it is then energetically favorable to have the species with the lowest cohesive energy at the surface, in order to minimize the energy cost due to lower coordination. In the present case, the nanoalloy clusters are formed by gas aggregation, in which $\mathrm{Na}$ and $\mathrm{K}$ atoms arrive randomly to the growing cluster. Reaching the observed surface-segregated core-shell structure requires sufficient intracluster diffusion. We cannot directly determine the temperature in the clusters; but in earlier studies, ${ }^{7}$ using the same setup, we could estimate the temperature of similarly produced free $\mathrm{Na}$ clusters to $\sim 100 \mathrm{~K}$ at the probing point where the cluster and the synchrotron-radiation beams intersect. This is also the temperature of the cryostat nozzle where the final cluster formation takes place. We see no reason why the NaK clusters would have a very different temperature. Such a low temperature will effectively preclude the diffusion required. In the primary aggregation phase, however, each aggregated atom releases cohesive energy to the growing cluster, implying that it is kept hot during the main aggregation process.
The hot random nanoalloy clusters gradually cool down in collisions with cold Ar atoms, yielding the observed cold surface-segregated core-shell structure. We conclude that the whole process allows sufficient intracluster diffusion to form the low-energy structures similar to those theoretically suggested-before the temperature gets too low. ${ }^{4}$

Concluding, we can state that, in the self-assembling gasaggregation process of binary nanoalloy formation out of the mixed-vapor phase of $\mathrm{Na}$ and $\mathrm{K}$, the element with the lower surface energy, K, occupies the surface sites. The surface segregation in the system makes Na practically absent on the surface, even when $\mathrm{K}$ is the minority species in the alloy core. For free clusters, similar effects have previously been observed for rare-gas systems. ${ }^{17,18}$ Strongly surfacesegregated structures may be rather common for free mixed clusters, and since the surface constitutes a major part of these nanoscale objects this segregation may have considerable consequences for their properties. This is important for technology-relevant metals and semiconductors, for chemical reactivity and catalytic activity, and for the systems of environmental interest such as certain molecular clusters. ${ }^{19}$

We would like to acknowledge the financial support from the Swedish Research Council (VR), the Göran Gustafsson Foundation, the Knut and Alice Wallenberg Foundation, the Crafoord Foundation, and the Swedish Foundation for Strategic Research (SSF). We would like to express our gratitude to J. Andersen for the fruitful discussion.

\footnotetext{
*Author to whom correspondence should be addressed.

${ }^{1}$ R. Ferrando, J. Jellinek, and R. L. Johnston, Chem. Rev. (Washington, D.C.) 108, 845 (2008).

${ }^{2}$ Wei-Heng Shih and D. Stroud, Phys. Rev. B 32, 804 (1985).

${ }^{3}$ D. J. González, L. E. González, and M. J. Stott, Phys. Rev. Lett. 94, 077801 (2005).

${ }^{4}$ A. Bol, G. Martin, J. M. López, and J. A. Alonso, Z. Phys. D: At., Mol. Clusters 28, 311 (1993).

${ }^{5}$ K. Sattler, J. Muehlbach, and E. Recknagel, Phys. Rev. Lett. 45, 821 (1980).

${ }^{6}$ C. Ellert, M. Schmidt, C. Schmitt, T. Reiners, and H. Haberland, Phys. Rev. Lett. 75, 1731 (1995).

${ }^{7}$ S. Peredkov, G. Öhrwall, J. Schulz, M. Lundwall, T. Rander, A. Lindblad, H. Bergersen, A. Rosso, W. Pokapanich, S. Svensson, S. L. Sorensen, N. Mårtensson, O. Björneholm, and M. Tchaplyguine, Phys. Rev. B 75, 235407 (2007).

${ }^{8}$ A. Rosso, G. Öhrwall, I. Bradeanu, S. Svensson, O. Björneholm, and M. Tchaplyguine, Phys. Rev. A 77, 043202 (2008).

${ }^{9}$ Handbook of Vapor Pressure, edited by C. L. Yaws (Gulf Publishing Company, Houston, TX, 1995), Vol. 4.

${ }^{10}$ D. M. Riffe, G. K. Wertheim, and P. H. Citrin, Phys. Rev. Lett. 67, 116 (1991).

${ }^{11}$ C. Nordling and J. Österman, Physics Handbook (Chartwell-
}

Bratt, Broomley, Kent, UK, 1980).

${ }^{12}$ K. Wong, G. Tikhonov, and V. V. Kresin, Phys. Rev. B 66, 125401 (2002)

${ }^{13}$ G. Makov and A. Nitzan, J. Chem. Phys. 88, 5076 (1988).

${ }^{14}$ P. M. Blass, X. L. Zhou, and J. M. White, Surf. Sci. 215, 74 (1989).

${ }^{15}$ J. J. Yeh and I. Lindau, At. Data Nucl. Data Tables 32, 1 (1985).

${ }^{16}$ M. Tchaplyguine, R. R. Marinho, M. Gisselbrecht, J. Schulz, N. Mårtensson, S. L. Sorensen, A. Naves de Brito, R. Feifel, G. Öhrwall, M. Lundwall, S. Svensson, and O. Björneholm, J. Chem. Phys. 120, 345 (2004).

${ }^{17}$ M. Tchaplyguine, M. Lundwall, M. Gisselbrecht, G. Öhrwall, R. Feifel, S. L. Sorensen, S. Svensson, N. Mårtensson, and O. Björneholm, Phys. Rev. A 69, 031201 (2004).

${ }^{18}$ M. Lundwall, W. Pokapanich, H. Bergersen, A. Lindblad, T. Rander, G. Ohrwall, M. Tchaplyguine, S. Barth, U. Hergenhahn, S. Svensson, and O. Björneholm, J. Chem. Phys. 126, 214706 (2007).

${ }^{19}$ M. Kulmala, I. Riipinen, M. Sipilä, H. E. Manninen, T. Petäjä, H. Junninen, M. Dal Maso, G. Mordas, A. Mirme, M. Vana, A. Hirsikko, L. Laakso, R. M. Harrison, I. Hanson, C. Leung, K. E. J. Lehtinen, V.-M. Kerminen, Science 318, 89 (2007). 